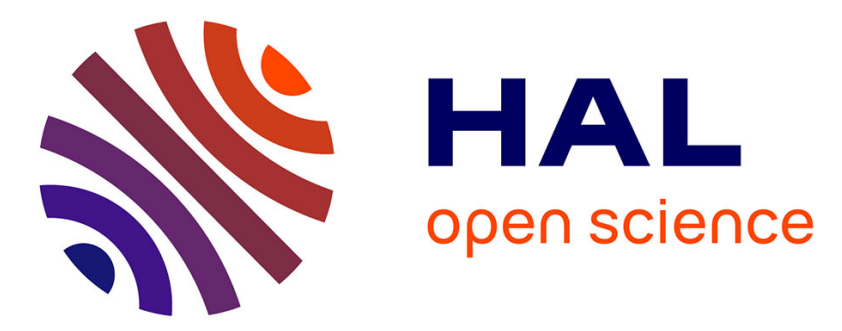

\title{
Road Network Resilience: How to Identify Critical Links Subject to Day-to-Day Disruptions
}

\author{
Pauline Gauthier, Angelo Furno, Nour Eddin El Faouzi
}

\section{To cite this version:}

Pauline Gauthier, Angelo Furno, Nour Eddin El Faouzi. Road Network Resilience: How to Identify Critical Links Subject to Day-to-Day Disruptions. Transportation Research Record, 2018, pp.1-12. 10.1177/0361198118792115 . hal-01945862

\section{HAL Id: hal-01945862 \\ https://hal.science/hal-01945862}

Submitted on 5 Dec 2018

HAL is a multi-disciplinary open access archive for the deposit and dissemination of scientific research documents, whether they are published or not. The documents may come from teaching and research institutions in France or abroad, or from public or private research centers.
L'archive ouverte pluridisciplinaire HAL, est destinée au dépôt et à la diffusion de documents scientifiques de niveau recherche, publiés ou non, émanant des établissements d'enseignement et de recherche français ou étrangers, des laboratoires publics ou privés. 


\section{Word Count:}

Number of words: 6684

Number of figures: 3 (250 words each)

Total: 7434

\title{
Road network resilience:
} how to identify critical links subject to day-to-day disruptions?

\author{
Camera-ready version uploaded on: Mars, $15^{\text {st }} 2018$
}

Pauline Gauthier, Master Student (Corresponding Author) ${ }^{1}$,

${ }^{1}$ Univ. Lyon, IFSTTAR, ENTPE, LICIT UMR_T9401, F-69675, Lyon, France pauline.gauthier@entpe.fr

Angelo Furno ${ }^{1}$,

${ }^{1}$ Univ. Lyon, IFSTTAR, ENTPE, LICIT UMR_T9401, F-69675, Lyon, France angelo.furno@ifsttar.fr

Nour-Eddin El Faouzi ${ }^{1,2}$

${ }^{1}$ Univ. Lyon, IFSTTAR, ENTPE, LICIT UMR_T9401, F-69675, Lyon, France

${ }^{2}$ Queensland University of Technology, STRC, Gardens Point Campus, 2 George Street, G.P.O. Box 2434, Brisbane, Queensland 4001, Australia. nour-eddin.elfaouzi@ifsttar.fr

Accepted for publication in the Transportation Research Record (TRR), Journal of the Transportation Research Board 


\begin{abstract}
Several disruptive events occur on road networks on a daily basis and affect traffic flow. Resilience analysis aims at assessing the consequences of such disruptions by quantifying the ability of a network to absorb and react to adverse events. In this paper, we advance a methodological approach based on resilience stress testing and a dynamic mesoscopic simulator. We aim at identifying and ranking the links most critical to the overall performance of the road network, taking into account dynamic properties of road traffic and focusing on day-to-day disruptions. As a metric to quantify road network performance in presence of such disruptions, we use the increase in overall travel cost. Then, we compare our approach with purely topological approaches.

We discuss the advantages and drawbacks of the different analyzed metrics. We prove that link ranking varies greatly depending on the metric. Specifically, the proposed stress testing methodology can produce very accurate results by taking into account demand and congestion, but requires computationally intensive simulations, being therefore prohibitive even on mediumsized networks. Conversely, purely static topological metrics can be inaccurate if they do not take into account traffic demand and network dynamics. Our study highlights the need for joining traditional traffic-agnostic topological resilience analysis with demand-aware dynamic stress-testing techniques.
\end{abstract}




\section{INTRODUCTION}

Roads tend to be disrupted by adverse weather conditions, human errors, or technological breakdowns. Such day-to-day disruptions affect traffic flow and may profoundly impair the ability of the transportation network to guarantee basic mobility services as well as emergency situation management, thus causing fundamental economic and social strains.

Resilience analysis aims at evaluating and predicting the consequences of such disruptions, and has become a crucial research concern recently $[1,2]$. In the field of road network, one possible approach consists in identifying the links that most strongly affect the overall performance. Operators and planners should be aware of the consequences deriving from reduced capacities on links. They should focus their efforts in improving and maintaining such critical links, since they may cause the most severe consequences on traffic operations when disrupted. Thus, a methodological approach and a set of resilience metrics are fundamental to identify critical links.

Similar to other complex systems, a city road transportation network can be modeled as a graph $G=(N, L)$ where road intersections are represented as nodes $(N)$ and roads as links $(L)$. This allows for exploiting graph theory and network connectivity analysis to study resilience. Graph theory can make resilience analysis very efficient in terms of computation costs, especially with the widespread adoption of big data technologies and cloud-computing. However, the phenomenon of congestion - dynamic, spatio-temporal, demand-dependent - is traditionally not addressed in topological studies on road traffic resilience $[3,4]$.

In this context, our work aims at answering the following research questions:

- How is it possible to identify the links that are most critical to the operation of the whole road network, especially with respect to day-to-day disruptions?

- Are topological metrics adequate to measure resilience for road networks?

- Which metrics are the most effective in assessing road network resilience, taking into account their dynamic, spatio-temporal and demand-dependent properties?

The main contributions of this paper are the following: (1) a methodology based on linkbased stress testing and a dynamic mesoscopic simulator is proposed for identifying critical links and quantifying road network robustness; (2) the methodology is compared to multiple topologybased metrics to clearly identify their limitations on a simplistic road network; (3) the approach is evaluated on a real-world network to identify and rank the most critical links in a realistic scenario; (4) guidelines are provided to decide when an approach can be preferred to the other one.

The paper is outlined as follows: Section 2 deals with related work. In Section 3, we review different resilience metrics and describe our methodological approach, by presenting the stress testing approach and our dynamic mesoscopic simulator. Section 4 describes our two case studies. Section 5 reports on the evaluation of our approach in the considered case studies. A final discussion is presented in Section 6, while some directions for further work are proposed in Section 7.

\section{LITERATURE REVIEW}

In this section, we firstly introduce the very general concept of resilience based on previous works, by adapting it to the context of road network analysis. Then, we present relevant road network resilience approaches for identifying critical links. 


\subsection{Resilience definition}

A wide diversity of definitions have been introduced in literature to characterize resilience. Woods identify four major concepts [5]: (1) resilience as rebound from trauma and return to equilibrium; (2) resilience as a synonym for robustness; (3) resilience as the opposite of brittleness, i.e., graceful extensibility when surprise challenges boundaries; (4) resilience as network architectures that can sustain the ability to adapt to future surprises as conditions evolve.

Bruneau et al. [6] define resilience as the ability to: (i) mitigating hazards (robustness or pre-perturbation resilience); (ii) containing the effects of disasters during they occurrence (reactivity); (iii) rapidly identifying occurred disruptions and mobilizing resources to quickly recover an acceptable traffic flow (recovery or post-perturbation resilience).

Sullivan et al. define in [7] road network robustness as the degree to which the network can function in the presence of capacity disruptions on links. A robust road network can face disruptions on links with only slight increases in overall network-wide travel costs. Conversely, a non-robust road network is subject to substantial increases in costs.

In the rest of the paper, we tackle the problem of resilience assessment according to the perspective provided by concept (2) in Woods [5], i.e., robustness, and the definition proposed in [7] by Sullivan et al. Our paper proposes an approach based on stress testing for assessing the impact of day-to-day disruptions on network links by measuring overall travel costs, aimed at quantifying the ability of the whole road network to absorb such an impact. We remark, as an additional contribution of our paper, that our approach also allows for resilience assessment under the occurrence of unexpected and possibly very rare events, which corresponds to concept (3) in Woods [5], i.e., graceful extensibility when surprise challenge boundaries. Our stress testing methodology is also applied for evaluating the impact of sudden travel demand variations on the overall travel costs.

\subsection{Resilience approaches}

The majority of the approaches aimed at quantifying resilience is based on topology models and static network-connectivity analysis $[8,3,4]$.

Among the plethora of metrics proposed to perform connectivity analysis, Betweenness Centrality (BC) is traditionally the best choice for traffic network analysis purposes, as it expresses the frequency with which a point falls between pairs of other points on the shortest paths connecting them [9]. BC has been therefore widely adopted to assess road resilience, by identifying topologically vulnerable links and intersections [10,11]. Very recently, approaches based on machine learning and big data solutions [12] have been proposed to significantly reduce computation time of $\mathrm{BC}$ on several kinds of very large complex networks [13].

Even though a large majority of studies focuses on static topological features of the network to identify its vulnerabilities, some authors have tried to join network topology features to more dynamic traffic-related information. Augmented definitions of BC take into account time-varying Origin-Destination (OD) travel demand $[14,15,16,17]$ as well as travel times $[16,18,19]$ for shortest paths computation. Such augmented metrics have been adopted for traffic flow analysis and prediction [14, 15, 18, 19], traffic assignment [16] as well as network performance monitoring in presence of extreme events [17]. To the best of our knowledge, none of the previously approaches has been evaluated with respect to day-to-day disruptions (i.e., reduced link capacity), which represents one of the core aspects of our paper. Also, their applicability to resilience assessment is still neither widespread nor fully understood, being these metrics often inaccurate in highly dynamic environments and prohibitive to compute on large scale networks [20, 12].

Another contribution of our paper is a methodology based on stress testing, i.e., pushing a 
system beyond its normal operational capacity and observing how it responds to the applied stress. Stress tests have been widely used in banking [21, 22], medical [23, 24] and hydro-geology domains [25]. Also referred to as network-disruption analysis, this approach has been occasionally leveraged in the field of transportation to identify critical links in a road network, but it is still at a very early stage [26, 27, 17]. Sullivan et al. [7] set different link-based capacity-disruption values for identifying and ranking the most critical links and quantifying road network resilience. Jenelius and Mattsson [28] developed the notion of Importance of a link, which is a function of the increase in travel time when the link is disrupted. Their method is demand-aware as they weight travel time by demand. Stress testing allows performing intra-network comparison: links are ranked based on their contribution to the overall network resilience $[29,30]$. Other authors have focused on identifying only the most critical nodes to be improved [30]. Some studies use probability-based models to calculate the likelihood that a network continues functioning after a given stress [31, 32]. To the best of our knowledge, the large majority of research works based on stress testing for assessing resilience of road network mainly deal with disasters and extreme events [17, 33, 34], instead of day-to-day disruptions as targeted in this paper.

\subsection{Assessment of the Literature}

The definition and quantification of resilience greatly vary depending on the context and application domain. No universal and totally agreed definition or metric of resilience exist.

Despite the plethora of work on resilience in various domains, a relatively small number of studies has targeted resilience of road networks explicitly, and very few applications on real world roads have been proposed. Our paper deals instead with a real network in the Paris agglomeration area, France.

The concept of resilience can be divided into two parts: pre-perturbation resilience (robustness), and post-perturbation resilience. In this study, focused on traffic modeling and analysis, we evaluate pre-perturbation resilience only. We do not account for post-perturbation socio-technical actions. As the aim of this paper is to rank and identify the most critical links in a given network with respect to day-to-day disruptions, we focus on intra-network comparisons.

In the literature, two general approaches are commonly used to quantify resilience [26]. The first is purely topological and usually demand-insensitive. The second takes into account traffic network performance and includes demand variability via simulation.

It is worth noting the lack of studies combining the stress-testing approach with the topological one. We analyze and compare the two different approaches to assess the resilience of road networks, and to determine their advantages and drawbacks in assessing road network resilience. For the first approach, i.e. the topological one, we consider and evaluate several metrics from graph-theory. As for the second approach, by means of a simulator, we inject perturbations in the network and quantify to what degree it can adjust to them.

\section{METHODOLOGY}

In the following, we report on the topological metrics considered in our analysis as well as the stress testing technique proposed to perform demand-aware, dynamic disruption analysis.

\subsection{Graph-theory metrics}

The topological metrics used in this study are based on Betweenness-Centrality (BC), originally proposed in [8]. BC measures the importance of the generic link $l$ of a graph by considering the 
1 number of shortest paths that traverse it, and is defined as follows:

$$
B C(l)=\sum_{i \neq l \neq j} \frac{\sigma_{i j}(l)}{\sigma_{i j}},
$$

2 where:

- $\sigma_{i j}(l)$ is the number of shortest paths from node $i$ to node $j$ that traverse link $l$;

- $\sigma_{i j}$ is the total number of shortest paths from node $i$ to node $j$.

In shortest path computation, links can be unweighted or weighted (e.g., in terms of the associated estimated travel time). In this study, we test and compare both cases. We also consider multiple variants of the $\mathrm{BC}$, reported in the following, to model different aspects of a road traffic network.

\subsubsection{BC for entry and exit nodes only}

We propose to use an alternative definition of $\mathrm{BC}$ consisting in calculating the shortest paths from entry to exit nodes only ${ }^{1}$. This definition introduces two advantages: computation time is reduced; the definition seems more realistic from a demand-aware perspective, since individuals tend to start and finish their trips over a subset of intersections. This corresponds to the OD representation of the traffic demand. The formula is the same as Eq. 1 with some exceptions:

- $i$ is selected from the entry-nodes subset, i.e., intersections used by vehicles to enter the network;

- $j$ is selected from the exit-nodes subset, i.e., intersections used by vehicles to leave the network.

In conclusion, we consider four different formulations of the BC:

- Unweighted BC (BC)

- Travel-time weighted BC (TTWBC)

- Unweighted BC on entry/exit nodes only (BC entries-exits)

- Travel-time weighted BC from entry to exit nodes only (TTWBC entries-exits)

Spatio-temporal traffic properties and phenomena, like demand, congestion and dynamic rerouting, are typically not addressed in graph-based models. Thus, graph-based metrics are usually incapable of capturing these aspects in turn.

\subsection{Demand-sensitive metric}

Jenelius et al. introduced in [2] the demand-aware metric of Importance (I) to characterize transportation vulnerability. This metric allows measuring network performance loss by using travel costs weighted by the traffic demand. Such metric is adequate for our methodological approach as it includes demand and the dynamic phenomenon of congestion (i.e., travel costs increase when

\footnotetext{
${ }^{1}$ The computation of betweenness centrality on a subset of nodes (entries and exits) is based on the function edge_betweenness_centrality_subset from the NetworkX Python library.
} 
traffic is congested). This metric uses a generic notion of travel cost, that can be specified depending on the study context and aim. In this paper, we define travel cost as travel time divided by travel distance (in seconds/kilometers). The importance of a link $l$ is the following:

$$
I(l)=\frac{\sum_{i} \sum_{j \neq i} x_{i j}\left(c_{i j}^{\delta}(l)-c_{i j}^{0}\right)}{\sum_{i} \sum_{j \neq i} x_{i j}},
$$

where:

- $x_{i j}$ is the demand from origin node $i$ to destination node $j$ (number of vehicles);

- $c_{i j}^{\delta}(l)$ is the mean travel cost from origin node $i$ to destination node $j$ when link $l$ is disrupted at level $\delta$;

- $c_{i j}^{0}$ is the mean travel cost from nodes $\mathrm{i}$ to $\mathrm{j}$ in the base case, i.e., without disruption

\subsection{Traffic model and algorithms}

To model traffic dynamics we use a dynamic mesoscopic simulator based on the Lighthill-WhithamRichards model [35, 36] and implemented in Matlab by our research group [37, 38].

The Lighthill-Whitham-Richards model is formulated in Lagrangian-space coordinates and uses both Lagrangian and Eulerian observations. It represents individual vehicles but only records their transit times at network nodes. A dynamic traffic assignment procedure distributes vehicles along all the possible alternative paths in the network, according to the traffic conditions at the moment the vehicle is generated. More precisely, travel times on all paths are calculated based on traffic flow, and the vehicle chooses the path that requires the smallest travel time. The following parameters have to be specified before running simulations: simulation duration, origin-destination demands and link capacities. This simulator is adequate for our stress testing approach: as opposed to static topological indicators, it includes traffic dynamic properties such as demand, congestion, trafficbased route assignment, dynamic shortest path computation and queues. Moreover, travel costs are calculated for each vehicle and can be easily extracted to compute network performance metrics, such as Importance from Eq. 2.

\subsection{Stress testing}

The aim of our stress-testing methodology is to identify the most critical links in the road network and to assess its resilience by considering the dynamic, spatio-temporal and demand-dependent properties of network traffic.

Stress testing can be leveraged for quantifying the adverse impacts associated to a reduction of capacity on specific links. Disruptive road events such as flooding, obstacles on the road, traffic accidents are likely to reduce the capacity of a given link and negatively affect network performance. Measuring network performance loss when reducing the capacity of a given link provides the criticality of this link to the operation of the whole network.

Therefore, stress testing is an adequate methodological approach to identify and rank the most critical links. It captures the relative importance of the disrupted link to the other links and assess the overall resilience of the whole road network from an intra-network comparison.

Our methodology for road network stress testing is composed of the following steps:

1. Simulating disruptive road events:

We propose two strategies to perform this step. In the first one, we simulate day-to-day road 
disruption as link capacity drops. The capacity-disruption level is defined as the reduction in link capacity, expressed as a fraction of the original one. In many studies, the capacitydisruption level is total, i.e. $100 \%$ of the original value, which means that the capacity of the link is reduced to 0 vehicles per hour [10]. The link is then completely removed from the road network. However, a 100\% capacity-disruption level does not accurately reflect the actual link capacity resulting from frequent day-to-day disruptions or minor events (e.g. number of lane reduction, adverse weather, etc.) that can affect the network. That is why we gradually reduce the capacity to analyze the evolution of the performance depending on the capacity-disruption level. We consider 5 possible capacity-disruption levels, denoted as $\delta$, on each examined link, i.e., $\delta \in\{0 \%, 20 \%, 40 \%, 60 \%, 80 \%\}$. Therefore, we can consider the following equation to compute the maximum capacity of the generic link $l$ in presence of a capacity-disruption level $\delta$ :

$$
\delta=100 \cdot\left(1-\frac{q_{\max }^{\delta}(l)}{q_{\max }^{0}(l)}\right)
$$

where:

- $\delta$ is the capacity-disruption level applied to $\operatorname{link} l$ (percentage) with $\delta \in\{0,20,40,60,80\}$;

- $q_{\text {max }}^{\delta}(l)$ is the capacity of link $l$ when it is disrupted at level $\delta$, (in vehicles/hours);

- $q_{\max }^{0}(l)$ is the capacity of link $l$ in the base case (in vehicles/hours);

As a second strategy to simulate disruptive road events, we consider increases in the traffic demand on specific entry/exit nodes of the network. By this approach, it is possible to simulate exceptional situations like city evacuations following extreme events (e.g., flooding, attacks, etc.) that typically put significant strain on the road infrastructure and result in total congestion. This strategy consists in changing the OD matrix, i.e., increasing the traffic flow from given entries, and comparing the stress testing results with another demand level.

Based on the selected strategy for disruptive road events, we set the parameters of our mesoscopic simulator (e.g., link capacity, traffic demand) and we simulate the network in the specific setting. For both strategies, travel costs are collected for all vehicles in order to compute the performance metrics described in the following point.

2. Computing overall performance loss:

This step is about quantifying the consequences of the simulated disruptive event on the operation of the whole network. To this purpose, we use the notion of road network performance, measured via the Importance metric of Eq. 2. Specifically, we consider travel time increase divided by travel distance as a measure of cost. Below, we provide the formulation of the overall performance loss $(P L)$, based on the notion of importance, when a link $l$ is disrupted:

$$
P L(l, \delta)=\sum_{v=1}^{n} \frac{c_{v}^{\delta}(l)-c_{v}^{0}}{n}
$$

where:

- $P L(l, \delta)$ is the overall performance loss when link $l$ is disrupted at level $\delta$ (seconds/kilometers);

- $c_{v}^{\delta}(l)$ is the travel cost of vehicle $v$ when link $l$ is disrupted at level $\delta$ (seconds/kilometers);

- $c_{v}^{0}$ is the travel cost of vehicle $v$ in the base case (seconds/kilometers); 
- $n$ is the number of vehicles in the network.

3. Analyzing the results:

For each link we know the performance loss corresponding to the considered capacity-disruption level (i.e., $0 \%, 20 \%, 40 \%, 60 \%$ and $80 \%$ of the original link capacity). As an example, $P L(2,40 \%)$ represents the overall performance loss on link 2 when it is disrupted at $40 \%$ of its initial capacity. In the evaluation section, we present and discuss the overall performance loss depending on the capacity-disruption level of the link according to two different strategies.

\subsection{Traffic robustness index}

The stress testing methodology reported in the previous section allows us to compute the overall performance loss for each link of the network with respect to five different capacity-disruption levels. To compare and identify critical links, we need a unique value of criticality associated to each link. To this purpose, a global metric is required to aggregate the performance loss values in the five different capacity-disruption levels. We propose the Stress Test Criticality metric (STC), defined as follows for the generic link $l$ :

$$
S T C(l)=\int_{\delta} P L(l, \delta),
$$

where:

- $S T C(l)$ is the Stress Test Criticality when link $l$ is stress-tested (seconds/kilometers)

- $\delta$ is the capacity-disruption level of link $l$ (percentage)

- $P L(l, \delta)$ is the overall performance loss (seconds/kilometers)

We use the trapezoid rule to approximate the integral in Eq. 5 from the (five) overall performance values computed on link $l$.

\section{CASE STUDIES}

The methodology and metrics described in the previous sections have been evaluated on two different case studies: the first one is related to a simple virtual network, used as a basic testbed for our approach; the second one is a real road network in France, which we use to confirm the validity of our results in a realistic scenario and to support the discussion on both simulation-based stress testing and topological metrics.

\subsection{A simple virtual road network}

This network is composed of 8 nodes ( 4 road intersections, 2 entries and 2 exits) and 9 links. The duration of each simulation is fixed to 10 minutes.

Figure 1a depicts the network structure with numbered links and flow directions. The network has been tested with two different demand levels reported as OD matrices in Figures 1b and 1c. 


\subsection{DIRIF: a real-world road network}

The DIRIF network is situated in South of Paris, France, and includes 868 links and 827 nodes (657 intersections, 86 entries and 84 exits). Its roads are mostly highways. Each simulation duration is fixed to 15 minutes. Since the network is much bigger than the one in Sec. 5.1 and traffic demand is extremely low on some links, we specify a higher simulation duration to ensure that enough vehicles travel through the whole network and to collect a significant number of travel cost observations. Simulation is performed with real demand data from 9:00 AM to 9:15 AM, i.e., the morning peaktime, in order to increase the probability of observing some performance loss in our stress tests. The network is graphically presented in Figures $3 \mathrm{a}$ and $3 \mathrm{~b}$.

\section{EVALUATION}

In this section, we present the results of our stress-testing methodology and discuss the link ranking derived from the different selected metrics. We show that the ranking of critical links on the same network can significantly vary when different indicators are used, thus proving that simple modifications of one centrality indicator can have a relevant impact on the capacity of the metric to capture different facets of resilience. Moreover, we discuss the advantages and drawbacks of each different approach in assessing road network resilience, and provide guidelines that can be helpful towards the definition of a new enhanced centrality metric.

\subsection{Application on a simple virtual network}

In the scenario of the simple network described in Section 4, we measured stress test criticality and all of the proposed topological metrics on all the links. To perform stress testing, we used both strategies described in Sec. 3.4, i.e., link capacity drop (referred as A in the following) and traffic demand increase (referred as B). The measures of stress-test criticality that result from the two strategies above are distinguished as STC A and STC B, respectively. STC A and B are calculated with the same formula, but different parameters are set before stress testing.

First we discuss the results of strategy A. In our simulations, we applied sequentially five capacity-disruption levels (i.e., $0 \%, 20 \%, 40 \%, 60 \%, 80 \%$ ) to each link. Then, we measured the network-wide performance loss (i.e., Eq. 4) consequent to the disruption applied to the link. The overall performance loss from our stress tests is reported on the y-axis of Figure 2a, while the corresponding capacity-disruption levels $(\delta)$ are reported on the x-axis. Results for different links are depicted with different colors and markers in the figure, using a linear interpolation. For readability, the figure only reports the five most critical links (i.e., those with the highest overall performance loss).

Intuitively, a link capacity drop translates into an increase of network-wide travel cost.

According to the definition provided in Eq. 5, the stress test criticality of a given link corresponds to the area below its curve. Thus, in strategy A, link 9 is the most critical to the operation of the whole road network, followed by links 5, 1, 2 and 8 . By using link-ranking from stress test criticality as a baseline, we compare in the following the other link rankings as derived from the different selected topological metrics. Figure 2c reports on such link rankings for both stress test criticality and the whole set of topological metrics.

As a preliminary consideration, it can be observed that the rankings of critical links on the same network may dramatically change depending on the metric, due to the different properties of the network captured by each of them. As an example, link 5 is on top of all the topological rankings whereas, with stress-test criticality, it is ranked second, below link 9. The top-rank of link 5 by all 
the topological metrics can be motivated considering the large number of shortest paths traversing this link: e.g., paths $(4,5),(7,5),(7,5,8),(7,5,9),(6,4,5),(7,5,8)$ are all shortest paths.

The different ranking issued by STC A can be easily explained. If link 5 is disrupted, alternative paths through link 2 exist for all individuals heading to exit 1 or exit 2. Conversely, when link 9 is disrupted, no alternative path exists for users willing to travel to exit 2 from both entry 1 and entry 2, thus resulting in important congestion and travel time increase for all individuals heading to exit 2. Additionally, traffic demand for exit 2 is very high (see OD matrix in Figure 1b). That explains why link 5 is more critical than link 9 in terms of topology, but less critical than link 9 when considering demand data, as made possible by our stress-testing methodology (based on dynamic simulations) and captured by the related criticality metric. This simple test clarifies how traditional demand-agnostic approaches may fail in properly ranking edge criticality.

Our simple test also shows that alternative paths may become shortest paths of the network as links are disrupted by adverse event, thus attracting traffic flow previously directed through the disrupted links. This represents another fundamental aspect that is impossible to capture with a static graph-based approach. However, this does not necessarily means that topological metrics are not good resilience indicators, but rather that road graph modeling should include a dynamic component (e.g., edge weights), and that betweenness centrality metrics should be rapidly re-computed after relevant network disruptions.

Another striking difference worth to analyze regards link 2: considered as one of the most critical ones according to the $\mathrm{BC}$ metric from entries to exits, it is the least critical one for the TTWBC and the TTWBC from entries to exits. The peculiarity of link 2 is its length: it is the longest one, thus demanding more time to be travelled than the other links. Metrics like BC and $\mathrm{BC}$ from entries to exits are not weighted, i.e., all links are valued equally, and are consequently unable to grasp this important aspect. Differently, links with high travel times are not considered critical by the analyzed weighted approaches, because they are not often part of shortest paths. The same consideration applies to link 7 , which is the second-longest link of the network.

Finally, it is worth noting that $\mathrm{BC}$ values are often clustered. In particular, the $\mathrm{BC}$ from entries to exits has many equal values and only 2 link ranks. Traditional topological metrics appear to have very limited capability to discriminate link criticality at a fine level. In this case, stress test criticality does not differentiate all links either, but this is due to capacity-disruption levels. For links 8, 6, 4, 7 and 3 which are all ranked at the $5^{\text {th }}$ place with the same value, both capacity-disruption and demand levels are not high enough to observe a significant performance loss compared to the base case. For example link 8 capacity disruption does not affect the overall network performance (see Figure 2a). The overall travel cost sticks to its base case value. That is why some links have the same criticality value. Then, stress test criticality differentiation between links depends on capacity-disruption and demand levels.

To further investigate this aspect, we use our second stress testing strategy B, i.e., with different traffic demand that is reported in Figure 1c. Results are shown in Figures 2b and 2c (i.e., STC B). Link ranking changes significantly when different demand levels are used. As an example, Link 8 becomes the most critical link, whereas in the previous case it involved no performance loss. This is due to the large increase in demand level associated to exit 1, which is directly connected to link 8 (see Figure 1a).

Takeaways: Critical link ranking is highly variable as different approaches are used. Resilience analysis via topological metrics is limited in the sense that such metrics do not usually take into account traffic demand and network re-configurations following disruptive events. Conversely, the simulation-based stress testing approach is able to capture these aspects thus providing more realistic rankings via the proposed performance loss metric. Stress testing can also be used to compare different road networks and sub-networks, by analyzing their response to similar stresses. Travel- 

time weighted $B C$ produces better estimations of link criticality with respect to unweighted $B C$, which
treats all links equally.

\subsection{Application on a real road network}

To confirm the results of our previous analysis in a realistic scenario, we considered Paris DIRIF road network, described in Section 4. Given the large size of this network and the high computation time associated to each network simulation ${ }^{2}$, it was prohibitive to perform an exhaustive stress-test analysis as in the simple network case. Therefore, we performed stress tests on a limited set of representative links: the three links with the highest demand, the three ones with the highest BC and three randomly selected edges with BC in three classes of values (high, medium and low). We discuss in the following only our simulations related to strategy $\mathrm{A}^{3}$. Figure $3 \mathrm{c}$ reports the actual values of the considered metrics for the analyzed links ${ }^{4}$.

Consistently with our previous analysis on the simple network, Figure 3c shows that rankings of critical links vary significantly. As an example, links 95, 93, 94 have a very high value of STC A, whereas the topological metrics rate them much less critical than links 802, 803, 60\%. As pointed out in the previous section, the STC A ranking appears to be more realistic since it captures the higher criticality of links 95, 93, 94 due to the associated higher demand (not reported due to space limitations).

On the small link subset considered in our analysis, taking into account travel times (TTWBC and TTWBC entries-exits) does not significantly change rankings, since link lengths (and therefore the average travel times) happen to be very similar on all considered links. Finally, it is worth noting that in the DIRIF network, BC values (especially in the entries/exits variations) tend to significantly cluster themselves (i.e., many edges have very similar values of BC), thus exhibiting a lower discriminant power than in the case considered in Section 5.1.

Takeaways: In a real-world scenario, stress testing proved to be a realistic and reliable approach to evaluate network resilience. Our evaluation confirms the importance of traffic demand and network dynamics for fine-grained ranking of the most vulnerable road-network links. Stress testing has however the drawback of requiring high execution times due to computationally intensive network simulations.

\section{DISCUSSION AND PERSPECTIVES}

From the previous results, we summarize in the following a few guidelines for properly characterizing critical links with respect to day-to-day disruptions, by means of an intra-network approach in different application contexts.

Firstly, if resilience has to be evaluated in a relatively static context (e.g., network maintenance or planning), BC and TTWBC appear to be adequate. In particular, if data about demand and travel times are not available, we recommend $\mathrm{BC}, \mathrm{BC}$ from entries and exits and $\mathrm{BC}$ on all paths from entries and exits. These indicators do not require special knowledge on network performance and demand data, but only the basic network topology (links and intersections). If traffic demand

\footnotetext{
${ }^{2}$ Stress testing one link from such a large network with 5 capacity drops takes more than 1 hour on an Intel Xeon E3 CPU equipped with 8 GB of RAM running a Matlab implementation of the mesoscopic simulator, properly configured to handle the DIRIF network in Scenarios A and B.

${ }^{3}$ Simulations for strategy $\mathrm{B}$ were in line with the results reported in the previous section and are not discussed due to space limitation.

${ }^{4}$ Differently from Figure 2c, we do not report metric rankings but actual values of the metrics for each analyzed link. This is motivated by the impossibility to get the full ranking for performance loss (i.e., STC).
} 
1 is the only missing information, Stress Test Criticality, TTWBC and TTWBC from entries to exits should be preferred, since they also take into account travel time information.

If the goal is instead to achieve a more accurate characterization of network resilience, stress testing should be chosen, since it produces reliable results by taking into account traffic demand and congestion phenomena. The drawback is that it requires many computationally intensive simulation, thus being recommended only in application scenarios that allow for larger computation time, or that address small-sized (sub-)networks. Conversely, in domains with very stringent requirements on response time (e.g., on-line vulnerability monitoring), topological indicators could be the only valuable option. However, it is worth to remark that efficient solutions are still required to compute these metrics on very large networks within reasonable computation time. For future work, we are currently working on the switch from a Matlab implementation of our simulationbased tools to a new implementation based on faster programming languages and approaches, i.e., explicitly designed for big data processing and real-time computation (e.g., Python and Scala on top of the Spark processing framework). In particular, we believe that implementing real-time advanced solutions for data-driven, on-line monitoring of road traffic resilience will constitute a fundamental research problem to investigate.

We advance that, in order to improve road network resilience analysis, future research work is needed that should consider joining graph-based approaches with demand-aware dynamic stresstesting techniques. In this context, we believe that a further improvement with topological metrics could be achieved by modeling the road network as a dynamic graph, whose link weights may change over time depending on actual traffic conditions and both structural and performancerelated network properties (e.g., road capacity, real-time traffic information, etc.).

Finally, we argue that future work should also consider area-wide disruptions in addition to single link-based disruptions, especially in the light of measuring the impact of extreme events.

\section{CONCLUSION}

Identifying critical links to the overall performance is part of road network resilience and intranetwork analysis. To this purpose, we have analyzed in this paper several topological metrics based on Betweenness-Centrality and proposed a stress testing approach exploiting a dynamic simulator. Stress testing appears to be a very promising solution for resilience analysis, allowing for measuring resilience in terms of the overall performance loss of the whole network consequent to simulated link disruptions.

Our analysis shows that link ranking varies greatly when different metrics are used. As opposed to purely topological metrics, the proposed stress-testing approach takes into account demand levels and dynamic characteristics of road traffic. However, it requires more computation time and data than traditional graph-based metrics. The choice of a relevant metric for assessing road network resilience should depend on the context and the specific application requirements.

Merging static topological metrics and demand-based approaches could be of further research interest. It could be relevant to adopt dynamic graphs modeling, using link weights to include dynamic information on the network. In such approach, topological metrics should be dynamically computed by means of efficient quasi real-time solutions. 


\section{DECLARATION}

2 The authors confirm contribution to the paper as follows: study conception and design: Gauthier, Furno, El Faouzi; data collection: Gauthier, Furno, El Faouzi; analysis and interpretation of results: Gauthier, Furno, El Faouzi; draft manuscript preparation: Gauthier, Furno, El Faouzi. All authors reviewed the results and approved the final version of the manuscript.

\section{References}

[1] Katja Berdica. An introduction to road vulnerability: what has been done, is done and should be done. Transport policy, 9(2):117-127, 2002.

[2] Erik Jenelius, Tom Petersen, and Lars-Göran Mattsson. Importance and exposure in road network vulnerability analysis. Transportation Research Part A: Policy and Practice, 40(7):537$560,2006$.

[3] M Di Gangi and AS Luongo. Measures of network vulnerability indicators for risk evaluation and exposure reduction. Environmental Health Risk III, 9:1151, 2005.

[4] Yingfei Tu, Chao Yang, and Xiaohong Chen. Methodology for evaluating and improving road network topology vulnerability. In Intelligent Computation Technology and Automation (ICICTA), 2010 International Conference on, volume 2, pages 664-669. IEEE, 2010.

[5] David D Woods. Four concepts for resilience and the implications for the future of resilience engineering. Reliability Engineering \& System Safety, 141:5-9, 2015.

[6] Michel Bruneau, Stephanie E Chang, Ronald T Eguchi, George C Lee, Thomas D OŔourke, Andrei M Reinhorn, Masanobu Shinozuka, Kathleen Tierney, William A Wallace, and Detlof Von Winterfeldt. A framework to quantitatively assess and enhance the seismic resilience of communities. Earthquake spectra, 19(4):733-752, 2003.

[7] JL Sullivan, DC Novak, L Aultman-Hall, and David M Scott. Identifying critical road segments and measuring system-wide robustness in transportation networks with isolating links: A linkbased capacity-reduction approach. Transportation Research Part A: Policy and Practice, 44(5):323-336, 2010.

[8] Linton C Freeman. A set of measures of centrality based on betweenness. Sociometry, pages 35-41, 1977.

[9] Yuanyuan Zhang, Xuesong Wang, Peng Zeng, and Xiaohong Chen. Centrality characteristics of road network patterns of traffic analysis zones. Transportation Research Record: Journal of the Transportation Research Board, 2256:16-24, 2011.

[10] David King, Amer Shalaby, and P Eng. Performance metrics and analysis of transit network resilience in toronto. In Transportation Research Board 95th Annual Meeting, number 16-2441, 2016.

[11] Bertrand Berche, Christian von Ferber, Taras Holovatch, and Yurij Holovatch. Resilience of public transport networks against attacks. THE EUROPEAN PHYSICAL JOURNAL B, 71(1):125-137, 2009. 
[12] Angelo Furno, Nour Eddin El Faouzi, Rajesh Sharma, and Eugenio Zimeo. Two-level clustering fast betweenness centrality computation for requirement-driven approximation. IEEE Big Data 2017 Conference, 2017.

[13] Angelo Furno, Nour-Eddin El Faouzi, Rajesh Sharma, and Eugenio Zimeo. Reducing pivots of approximated betweenness computation by hierarchically clustering complex networks. In International Conference on Complex Networks and their Applications, pages 65-77. Springer, 2017.

[14] Amila Jayasinghe, Kazushi Sano, and Hiroaki Nishiuchi. Explaining traffic flow patterns using centrality measures. International Journal for Traffic \& Transport Engineering, 05(02):134$149,2015$.

[15] Aisan Kazerani and Stephan Winter. Modified betweenness centrality for predicting traffic flow. 2, 2009.

[16] Rami Puzis, Yaniv Altshuler, Yuval Elovici, Shlomo Bekhor, Yoram Shiftan, and Alex Pentland. Augmented betweenness centrality for environmentally aware traffic monitoring in transportation networks. Journal of Intelligent Transportation Systems, 17(1):91-105, 2013.

[17] Wisinee Wisetjindawat, Amirhassan Kermanshah, Sybil Derrible, and Motohiro Fujita. Stochastic modeling of road system performance during multihazard events: Flash floods and earthquakes. Journal of Infrastructure Systems, 23(4):04017031, 2017.

[18] Yaniv Altshuler, Rami Puzis, Yuval Elovici, Shlomo Bekhor, and Alex BaglioniPentland. Augmented betweenness centrality for mobility prediction in transportation networks. Finding Patterns of Human Behaviors in Network and Mobility Data (NEMO), 2011.

[19] Yew-Yih Cheng, Roy Ka-Wei Lee, Ee-Peng Lim, and Feida Zhu. Measuring centralities for transportation networks beyond structures. In Applications of Social Media and Social Network Analysis, pages 23-39. Springer, 2015.

[20] Yingying Duan and Feng Lu. Robustness of city road networks at different granularities. Physica A: Statistical Mechanics and its Applications, 411:21-34, 2014.

[21] Andrew Haldane. Why banks failed the stress test. BIS Review, 18:2009, 2009.

[22] Til Schuermann. Stress testing banks. International Journal of Forecasting, 30(3):717-728, 2014.

[23] NORA Goldschlager, Artur Selzer, and Keith Cohn. Treadmill stress tests as indicators of presence and severity of coronary artery disease. Ann Intern Med, 85(3):277-286, 1976.

[24] Steven W Baertschi, Karen M Alsante, and Robert A Reed. Pharmaceutical stress testing: predicting drug degradation. CRC Press, 2016.

[25] Limin M Zhang, Liang Gao, Shengyang Y Zhou, Raymond WM Cheung, and Suzanne Lacasse. Stress testing framework for managing landslide risks under extreme storms. In Workshop on World Landslide Forum, pages 17-32. Springer, 2017.

[26] Lars-Göran Mattsson and Erik Jenelius. Vulnerability and resilience of transport systems-a discussion of recent research. Transportation Research Part A: Policy and Practice, 81:16-34, 2015. 
[27] Julie Clarke and Eugene Obrien. A multi-hazard risk assessment methodology, stress test framework and decision support tool for transport infrastructure networks. Transportation Research Procedia, 14:1355-1363, 2016.

[28] Erik Jenelius and Lars-Göran Mattsson. Road network vulnerability analysis of area-covering disruptions: A grid-based approach with case study. Transportation research part A: policy and practice, 46(5):746-760, 2012.

[29] Darren M Scott, David C Novak, Lisa Aultman-Hall, and Feng Guo. Network robustness index: A new method for identifying critical links and evaluating the performance of transportation networks. Journal of Transport Geography, 14(3):215-227, 2006.

[30] Katja Berdica and Lars-Göran Mattsson. Vulnerability: a model-based case study of the road network in stockholm. Critical infrastructure, pages 81-106, 2007.

[31] Zhen-Ping Du and Alan Nicholson. Degradable transportation systems: sensitivity and reliability analysis. Transportation Research Part B: Methodological, 31(3):225-237, 1997.

[32] Richard Church and M Paola Scaparra. Analysis of facility systemsŕeliability when subject to attack or a natural disaster. Critical Infrastructure, pages 221-241, 2007.

[33] Brian Donovan and Daniel B Work. Using coarse gps data to quantify city-scale transportation system resilience to extreme events. arXiv preprint arXiv:150\%.06011, 2015.

[34] Mayada Omer, Ali Mostashari, and Roshanak Nilchiani. Assessing resilience in a regional roadbased transportation network. International Journal of Industrial and Systems Engineering, 13(4):389-408, 2013.

[35] Michael J Lighthill and Gerald Beresford Whitham. On kinematic waves. ii. a theory of traffic flow on long crowded roads. In Proceedings of the Royal Society of London A: Mathematical, Physical and Engineering Sciences, volume 229, pages 317-345. The Royal Society, 1955.

[36] Paul I Richards. Shock waves on the highway. Operations research, 4(1):42-51, 1956.

[37] Aurélien Duret, Ludovic Leclercq, and Nour-Eddin El Faouzi. Data assimilation using a mesoscopic lighthill-whitham-richards model and loop detector data: Methodology and largescale network application. Transportation Research Record: Journal of the Transportation Research Board, (2560):26-36, 2016.

[38] Jorge A Laval and Ludovic Leclercq. The hamilton-jacobi partial differential equation and the three representations of traffic flow. Transportation Research Part B: Methodological, 52:17-30, 2013. 


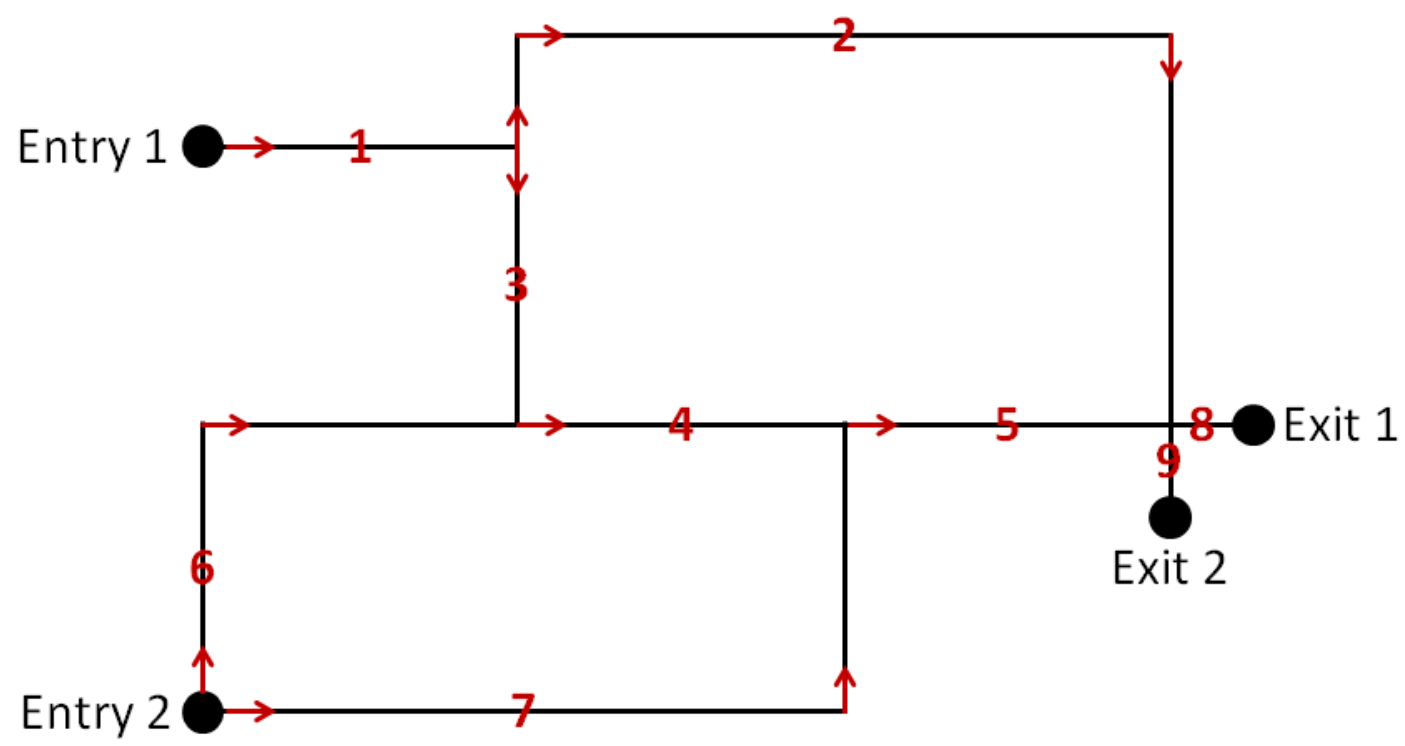

(a) Simple virtual road network

\begin{tabular}{|c|c|c|}
\hline Origin $\quad$ Destination & Exit 1 & Exit 2 \\
\hline Entry 1 & 375 & 375 \\
\hline Entry 2 & 250 & 1000 \\
\hline
\end{tabular}

(b) OD demand (vehicles/hour) for the simple virtual network (strategy A)

\begin{tabular}{|c|c|c|}
\hline Origin $\quad$ Destination & Exit 1 & Exit 2 \\
\hline Entry 1 & 500 & 500 \\
\hline Entry 2 & 1200 & 300 \\
\hline
\end{tabular}

(c) OD demand (vehicles/hour) for the simple virtual network in (strategy B)

Figure 1: Simple virtual network. Network structure (a) and OD travel demand (b, c) for the two considered strategies. 


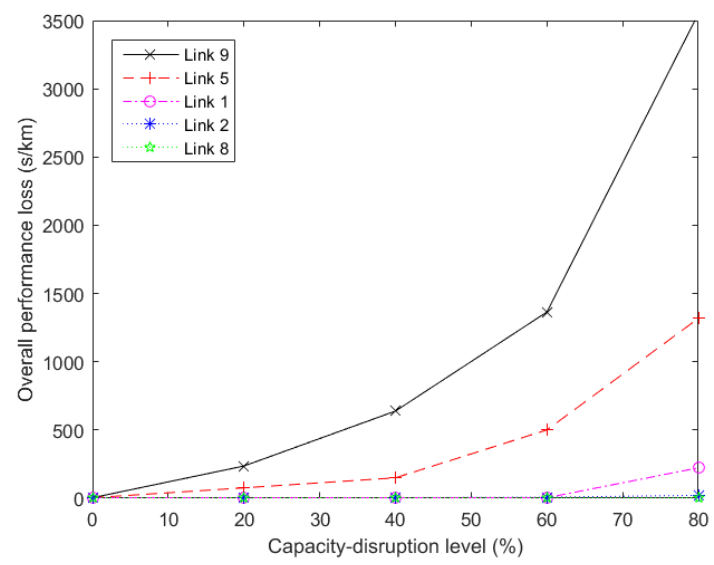

(a) Overall Performance Loss, strategy A (STC A)

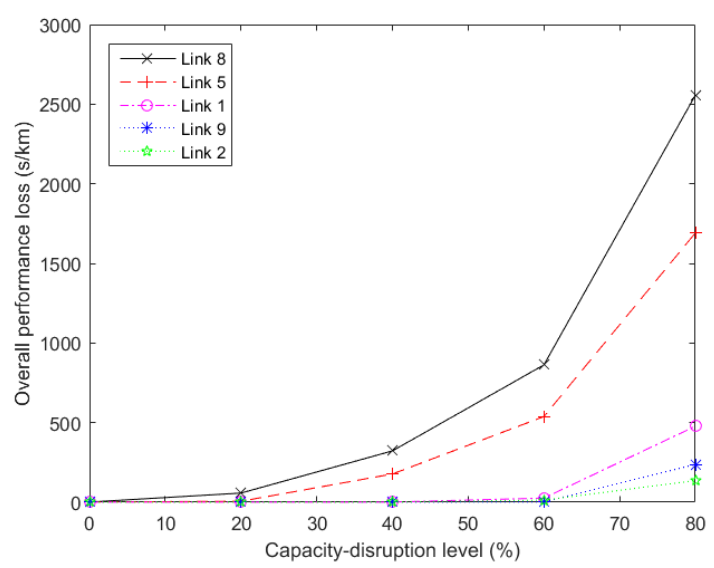

(b) Overall Performance Loss, strategy B (STC B)

\begin{tabular}{|c|c|c|c|c|c|c|}
\hline Link & STC A & STC B & BC & $\begin{array}{c}\text { BC } \\
\text { entries-exits }\end{array}$ & TTWBC & $\begin{array}{c}\text { TTWBC } \\
\text { entries-exits }\end{array}$ \\
\hline \hline $\mathbf{9}$ & $1^{\text {st }}$ & $4^{\text {th }}$ & $2^{\text {nd }}$ & $1^{\text {st }}$ & $4^{\text {th }}$ & $3^{\text {rd }}$ \\
\hline $\mathbf{5}$ & $2^{\text {nd }}$ & $2^{\text {nd }}$ & $1^{\text {st }}$ & $1^{\text {st }}$ & $1^{\text {st }}$ & $1^{\text {st }}$ \\
\hline $\mathbf{1}$ & $3^{\text {rd }}$ & $3^{\text {rd }}$ & $2^{\text {nd }}$ & $1^{\text {st }}$ & $4^{\text {th }}$ & $3^{\text {rd }}$ \\
\hline $\mathbf{2}$ & $4^{\text {th }}$ & $5^{\text {th }}$ & $2^{\text {nd }}$ & $1^{\text {st }}$ & $7^{\text {th }}$ & $5^{\text {th }}$ \\
\hline $\mathbf{8}$ & $5^{\text {th }}$ & $1^{\text {st }}$ & $2^{\text {nd }}$ & $1^{\text {st }}$ & $4^{\text {th }}$ & $3^{\text {rd }}$ \\
\hline $\mathbf{6}$ & $5^{\text {th }}$ & $6^{\text {th }}$ & $4^{\text {th }}$ & $2^{\text {th }}$ & $5^{\text {th }}$ & $4^{\text {th }}$ \\
\hline $\mathbf{4}$ & $5^{\text {th }}$ & $7^{\text {th }}$ & $2^{\text {nd }}$ & $2^{\text {th }}$ & $2^{\text {nd }}$ & $2^{\text {nd }}$ \\
\hline $\mathbf{7}$ & $5^{\text {th }}$ & $8^{\text {th }}$ & $3^{\text {th }}$ & $1^{\text {st }}$ & $6^{\text {th }}$ & $4^{\text {th }}$ \\
\hline $\mathbf{3}$ & $5^{\text {th }}$ & $8^{\text {th }}$ & $3^{\text {th }}$ & $2^{\text {th }}$ & $3^{\text {rd }}$ & $3^{\text {rd }}$ \\
\hline
\end{tabular}

(c) Simple network link rankings generated by the different metrics of criticality

Figure 2: Performance evaluation on the simple virtual network 


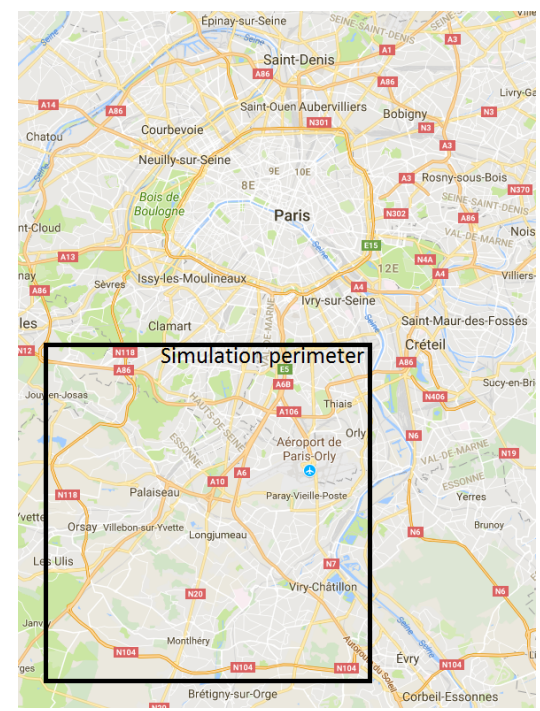

(a) DIRIF, geographic location

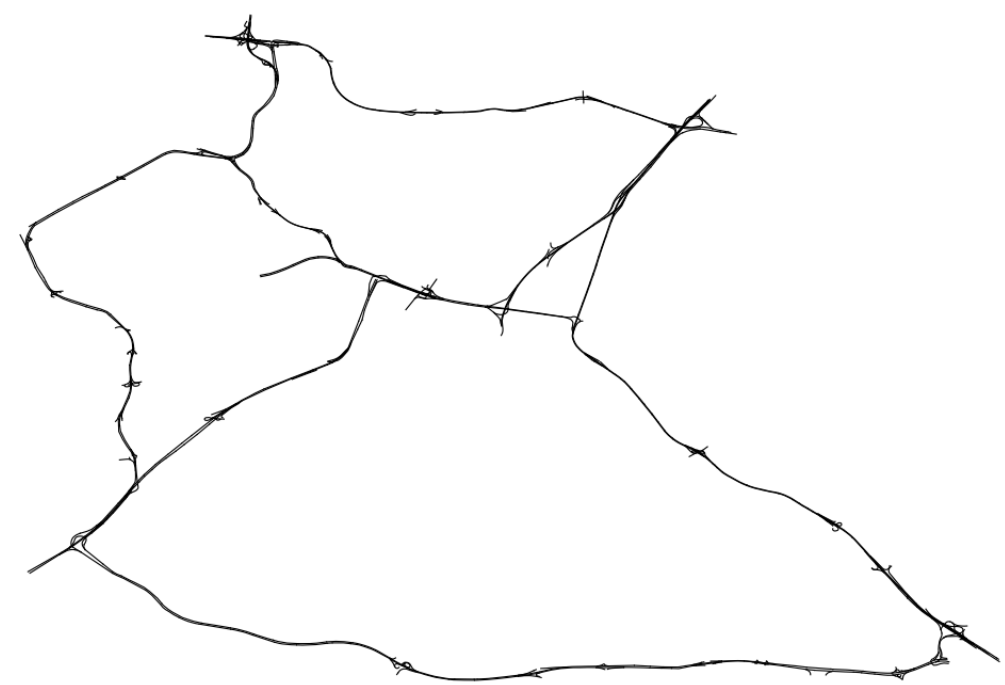

(b) DIRIF network

\begin{tabular}{|c|c|c|c|c|c|c|}
\hline \multirow{4}{*}{$\begin{array}{l}\text { Highest- } \\
\text { demand } \\
\text { links }\end{array}$} & Link & STC A & BC & $\begin{array}{c}\mathrm{BC} \\
\text { entries-exits }\end{array}$ & TTWBC & $\begin{array}{c}\text { TTWBC } \\
\text { entries-exits }\end{array}$ \\
\hline & 95 & 117 & 2628 & 83 & 2628 & 83 \\
\hline & 93 & 102 & 660 & 83 & 660 & 83 \\
\hline & 94 & 101 & 1974 & 83 & 1974 & 83 \\
\hline \multirow{6}{*}{$\begin{array}{c}\text { Highest- } \\
\text { BC } \\
\text { links }\end{array}$} & 802 & $\overline{42}$ & 192497 & 3029 & 192717 & 3039 \\
\hline & 803 & 27 & 192521 & 3029 & 192741 & 3039 \\
\hline & 607 & 27 & 192509 & 3029 & 192729 & 3039 \\
\hline & 608 & 44.6 & 192449 & 3029 & 192669 & 3039 \\
\hline & 397 & 15.8 & 83164 & 1139 & 83164 & 1139 \\
\hline & 672 & 14.5 & 10 & 1 & 10 & 1 \\
\hline
\end{tabular}

(c) DIRIF network link values generated by the different metrics of criticality

Figure 3: The DIRIF road network in Paris agglomeration (a) and (b). Evaluation results (c). 